\title{
Enzymes by Evolution: Bringing New Chemistry to Life*
}

\author{
Frances H. Arnold ${ }^{1}$
}

\begin{abstract}
Not satisfied with nature's vast enzyme repertoire, we want to create new ones and expand the space of genetically encoded enzyme functions. We use the most powerful biological design process, evolution, to optimize existing enzymes and invent new ones, thereby circumventing our profound ignorance of how sequence encodes function. Mimicking nature's evolutionary tricks and using a little chemical intuition, we can generate whole new enzyme families that catalyze important reactions, including ones not known in biology. These new capabilities increase the scope of molecules and materials we can build using biology.
\end{abstract}

Keywords: Enzyme Evolution; Directed Evolution; Carbene Transfer; Organosilicon; Biocatalysis.

I want to explore biology beyond the natural world, using evolution as a tool to create things that nature has not made. Thus this talk is about bringing new chemistry to life, making enzymes that catalyze reactions we have not (yet) found in nature. You might think there is already enough interesting chemistry out there in the biological world to keep us busy and satisfied. I will argue, however, that human chemists have been pretty clever over the last one hundred years, discovering catalysts and making useful molecules, and it would be marvelous to be able to genetically encode some of their inventions, or, even better, go beyond what both humans and nature have been able to.

I started my career as an engineer: I have a degree in mechanical and aerospace engineering, and I worked at the Solar Energy Research Institute, newly formed when Jimmy Carter was president. In the late 1970 s, my country actually had a national goal of 20 percent renewable energy by the year 2000. Sadly, we didn't stick to that. When the election of Ronald Reagan forced me to rethink my plans in solar energy, I went to graduate school for a $\mathrm{PhD}$ in chemical engineering. My timing was perfect: I was at U.C. Berkeley at the beginning of the DNA revolution. Imagine how exciting it was to be a student when brand-new companies like Genentech, Chiron, and Amgen were starting up, all built on the idea that we could manipulate the biological world at the level of DNA and rewrite the code of life to solve human problems. I was inspired by this remarkable technology to dream of new ways to make the materials, fuels, and chemicals that we need in our daily lives.

The more I learned about enzymes, the more impressed I was by how these amazing biomolecules take materials and energy from the environment and convert them into self-repairing, self-reproducing, responsive, adaptive... life. Enzymes are the most beautiful of all the molecules and are so well designed by evolution to do chemistry better than any human. When it comes to catalysis, there's nothing that can beat an enzyme.

If we could rewrite the code of life, or even just the code of enzymes, then we could look at an organism, say a yeast or a bacterium, as a chemical factory that uses renewable resources and converts those into valuable products, all programmed in the DNA. There's the ultimate, programmable, green chemical synthesis machine! This is a vision that has been around for 30 years or more. It's been hard to implement, though, because it's very hard to compete with

\footnotetext{
${ }^{1}$ Division of Chemistry and Chemical Engineering, California Institute of Technology, Pasadena, CA 91125, USA. Published online 22 July 2018; doi:10.1142/S2529732518400023

*This article was taken from a presentation delivered by Dr. Arnold at the Molecular Frontiers Symposium on Tailored Biology at the Royal Swedish Academy of Sciences, May 2017. It is published here in MFJ with permission from Dr. Arnold.

This is an Open Access article published by World Scientific Publishing Company. It is distributed under the terms of the Creative Commons Attribution 4.0 (CC-BY) License. Further distribution of this work is permitted, provided the original work is properly cited.
} 
AATAGCCGTTATTTCCGGATGTGCATA

GCTGATTTGACCCATCCGGTACACCA

ATDACAAATCCCGATTTGATCGTGTGC GCGACATGTCTTCCGGCGACACATGT GTCTCTCACTCCGAGAGATCGGTTAG AGTCTCGGTTAACCACACGTCCCGGA TATATTTAATTGGCCGGAGAGTCTCCC GCGCGACATAAGGAGTCCTCGTTTCG AGATACGTACGGCATGGTGACACCAG TTGCCCTCTGATTCCCGGAGCCTCTTT GAAAACGTCGAGTCGAATCGAAGTTC GAACCCCGGATCGGGTCCACCAACTT AGAGATGTGTGTGCGCTGACTCAGTC ATTITGACCCATCCGGTACACCAATGG GTCGGACAAATCCCGATTTGATCGTGT GCGCGACATGTCTTCCGGCGACACAT GTGTCTCTCACTCCGAGAGATCGGTT AGAGTCTCGGTTAACCACACGTCCCG GATATATTTAATTGGCCGGAGAGTCTC CAATAGCCGTTATTTCCGGATGTGCAT AGCTGATTTGACCCATCCGGTACACC AATGGGTCGGACAAATCCCGATTTGA TCGTGTGCGCGACATGTCTTCCGGCG ACACATGTGTCTCTCACTCCGAGAGA

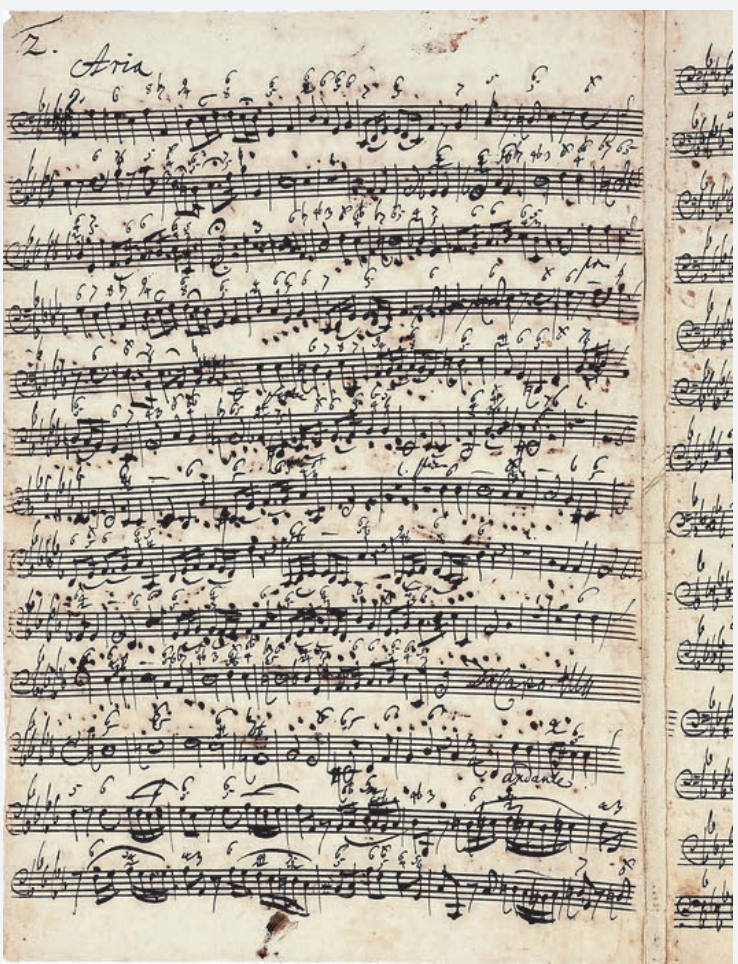
TCGGTTAGAGTCTCGGTTAACCACAC

Directed evolution is a molecular optimization process on a multi-dimensional fitness landscape, where fitness is performance, defined by the user.
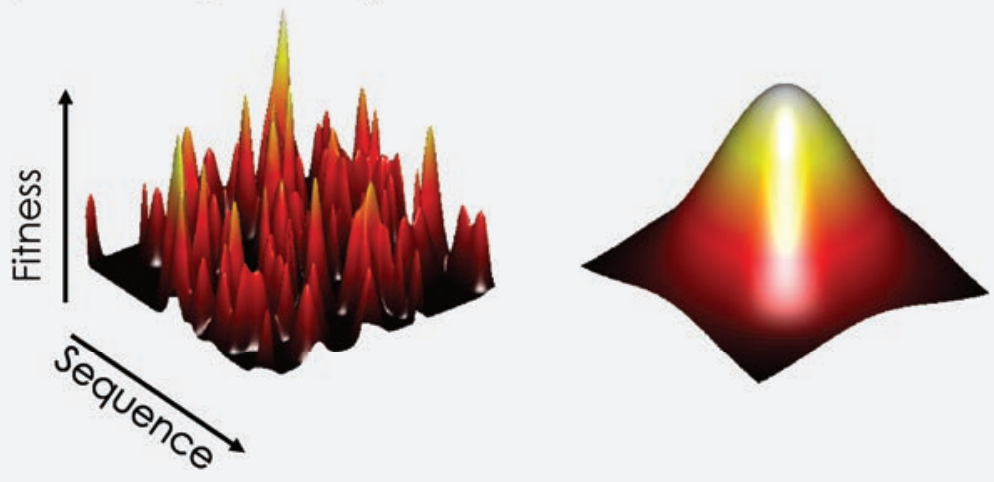

'make more microbes' and instead have a microbe make the chemicals that we want. That's a very difficult DNA design problem.

And of course my colleagues, who are pretty good chemists, say, "Oh, Frances, you know biology is great, has lots of interesting chemistry, but it can't do what I can do." And they are right, because the chemical industry is based on chemistry, not biology. Ours is a synthetic world, full of products made by synthetic chemistry, containing molecules and chemical bonds you don't find in the biological world. Biology can do some of the things that human

pumping oil out of the ground and using that as a feedstock for fuels and chemicals. But if we want sustainable ways of making these critical substances, we have to be able to compete. That means we have to compose new DNA so well that we can subvert three-plus billion years of evolution that says chemists have come up with, but many others have no biological precedent.

So here's what I want to do about it: I want to be able to genetically encode chemistry that has been invented by human beings. And not only that, I want to go beyond where 


\section{Directed evolution exploits smooth paths in the fitness landscape.}

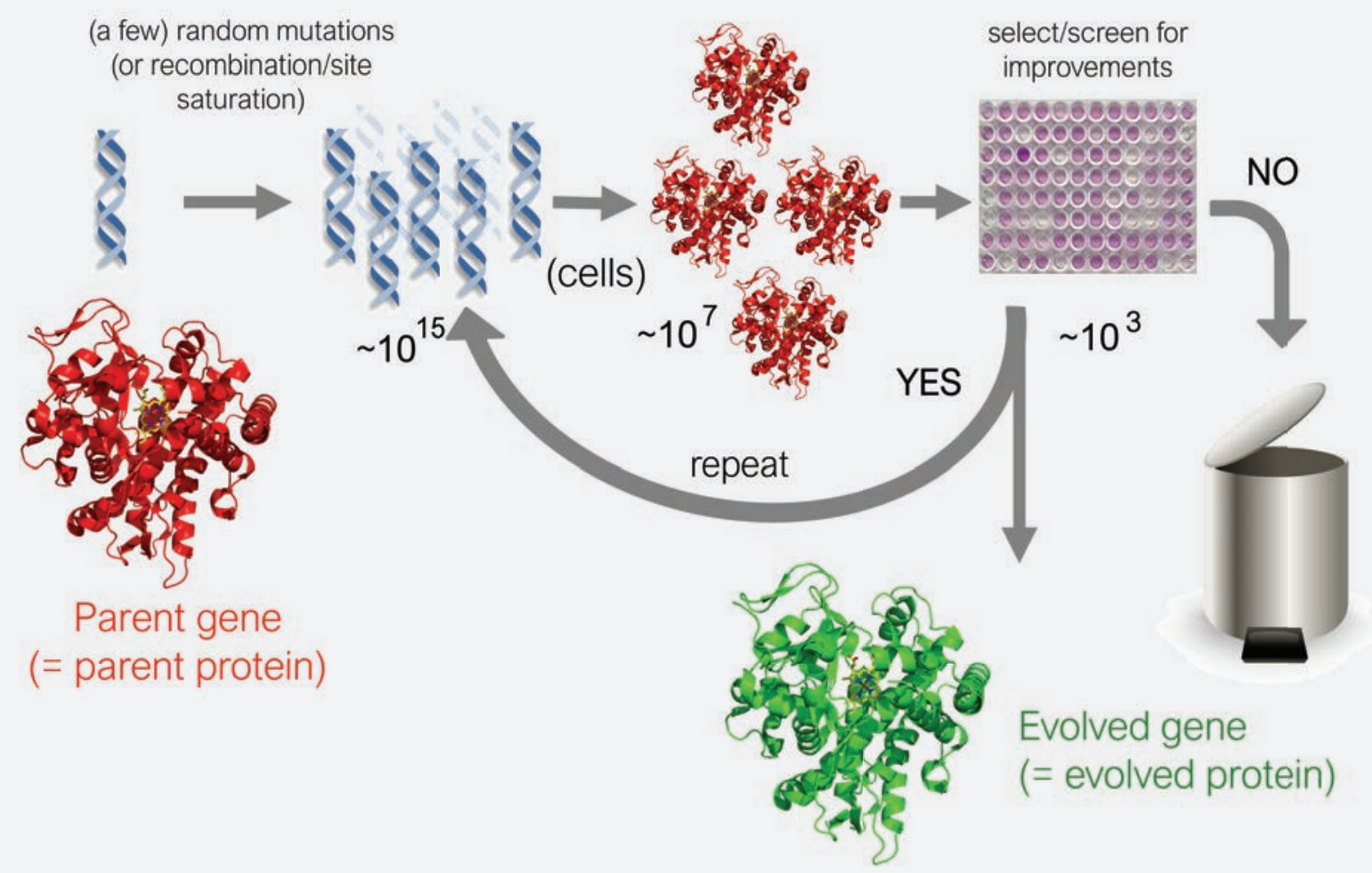

Novelty is already there. Enzymes are 'promiscuous' and can catalyze new reactions (albeit at a low level).

Access to new functions relies on some (even very small) overlap that can be drawn out and optimized during evolution.

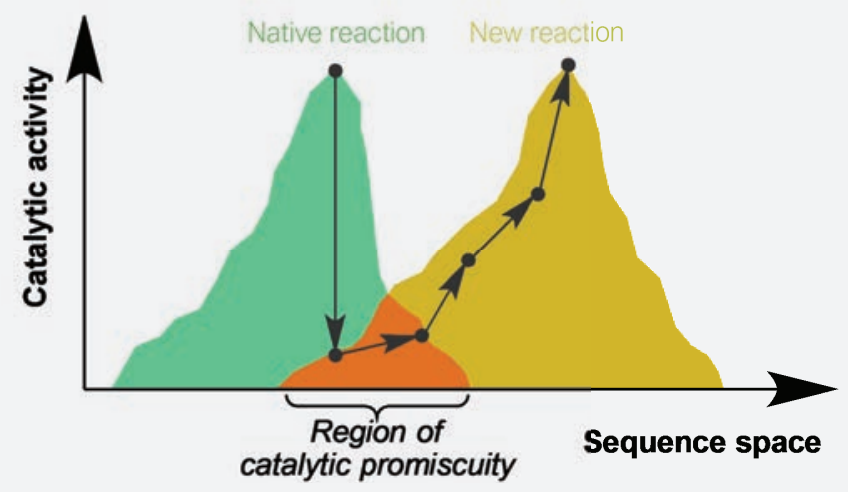

not already exist in nature-has been around for as long as we have been able to manipulate DNA. Progress in creating new enzymes has been stymied by two major hurdles on the path between a DNA sequence and what the resulting protein does. First, how does the sequence encode a folded protein? The field has been making good headway on this 'protein folding' problem, but in fact that's not the problem anybody cares about in my business, because we are all focused on the more important issue of how a sequence encodes function. And that function needs to be good enough to compete with pumping oil out of the ground or at least your favorite synthetic chemist. That's a very high bar. Thirty years ago people said humans have been able to go chemically and do it in a sustainable fashion. This hard. The enzyme design problemthat is, how you design a good enzyme catalyst that does that they would have this problem solved in 5 years, and it still hasn't been solved today. Thus when I started out as a protein engineer, I had to take a different tack, not the one all 


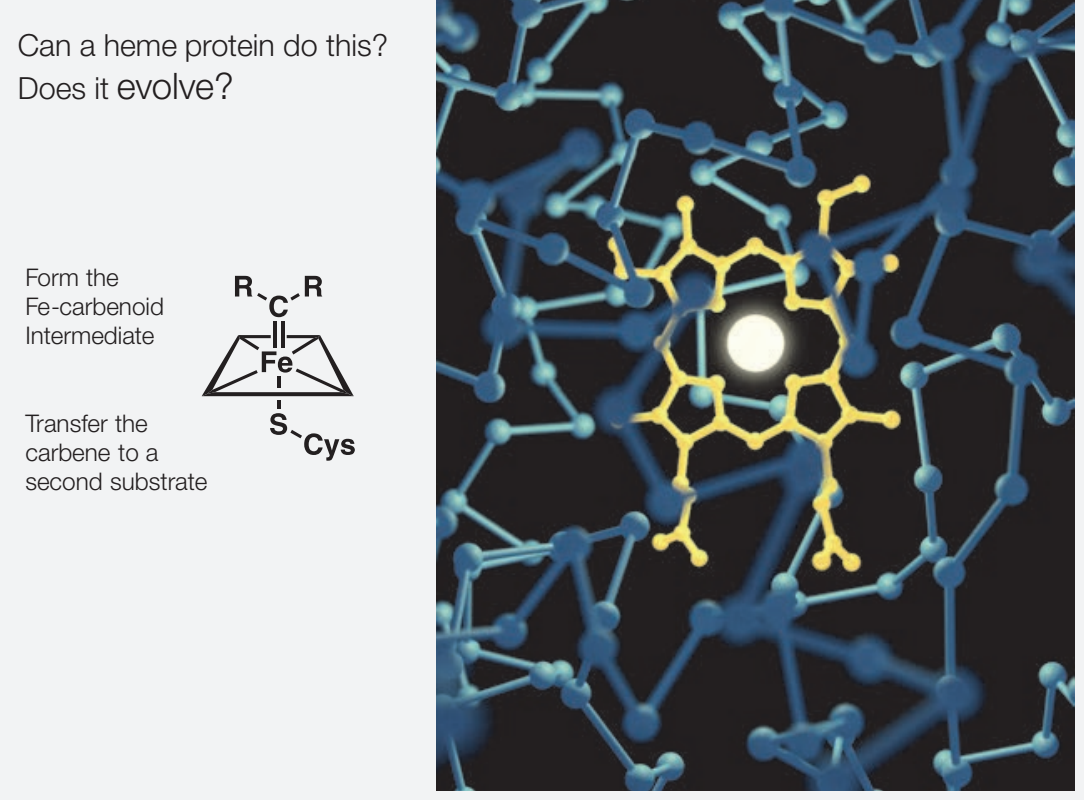

the bottom of your shoe and that are better than anything a human can design.

In fact, we would not be here unless evolution did a very good job at making all those wonderful proteins that we can use, enjoy, and even hope to make better. If you look at the sequences of homologous enzymes that exist today, you can build their family trees and see how they came from a common ancestor through a process of mutation and selection. This simple algorithm of evolution created all of these proteins so beautifully tuned for their biological roles. $\mathrm{Na}-$ ture figured it out. So I asked, why not use this design algorithm to build new enzymes?

The heme protein is a self-assembling, DNA-encoded, chiral metal complex whose structure and electronic properties can be tuned by mutation.

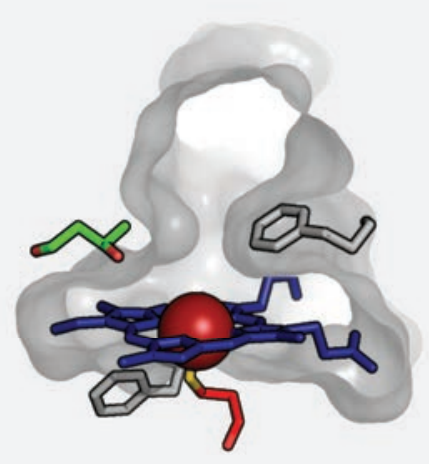

the protein engineers were using, which was to get a crystal structure of your protein, use your big brain to identify where you're going to make changes, make those changes, and take two months to find out it doesn't work.

The technology of molecular biology has progressed tremendously in the past 30 years. You can now synthesize in the test tube essentially any DNA you want. You can type your sequence into your computer, e-mail it to your favorite supplier, and in a few days or weeks you will get in the mail the actual DNA. It's amazing. So now we can read DNA, we can write DNA, and we can edit DNA. But no one knows how to compose DNA. No designer did it: three billion years of evolution gave us these wonderful catalysts that you can scrape off
This is not a new idea, at least in part. I want to use evolution for forward engineering, using all the new tools of molecular biology. But humans have been manipulating DNA, rewriting the code of life, for thousands of years by choosing who goes on to parent the next generation. We've been making corn, carrier pigeons, lab rats, race horses, you name it, we have been manipulating the biological world at the level of DNA to solve human problems, such as how do you feed a population and have time left over to do science? We have been breeding organisms that help us or that merely give us pleasure, and these are not remotely 'natural.' This poodle would not be here without the intervention of human beings. If it were to be released in the wild, at least in my city, it would be eaten by the coyotes. We have been using this process of breeding and artificial selection effectively and to our benefit for quite a while, all without understanding the details of how sequence codes function.

Now, of course, this is limited to evolution and breeding of the biological world, where the sequence diversity is limited. Worms mate with worms, monkeys with monkeys, but you don't cross the two. Maybe you can increase your random mutations by taking more airplane flights or smoking a few more cigarettes, but you can't really control the mutation load. But remember, I said you can now create any DNA sequence you want. With 'molecular breeding' you can choose 3 parents or 33 parents, and you can dial in any level of mutation. You become the breeder of molecules and have much more control, for good or for bad, over this process. The problem is that we have all this control, but we don't know the rules. You might think it's a hard problem, because for even a single 
Selective cyclopropanation at scale: replace precious metal catalyst and multi-step synthesis with a single step that uses Fe-based catalyst<smiles>C=C(C=[N+]=N)OCC</smiles>

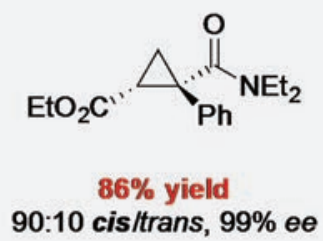

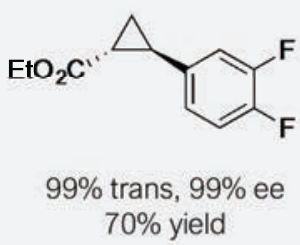

$70 \%$ yield protein a few hundred amino acids long and 20 amino acids to choose from at each positions, that's a really big space of possible sequences. It's bigger than the number of particles in the universe. It's bigger than the national debt of the United States. It's a really big number, and it's mostly empty: most of those sequences don't encode anything that solves a human problem, and they certainly don't compete with pumping oil out of the ground. How do you search a space of enzymes that's bigger than you can even begin to comprehend and mostly empty? It might look like an insurmountable optimization problem until you think a little more about the nature of the fitness landscape. If the landscape is smooth, you can take a random uphill walk to the top, but if it's very rugged, every time you make a mutation you fall off into the abyss. John Maynard Smith thought about this problem back in 1970 in my favorite, two-paged Nature paper. And this was before all those sequences were available to help us to see how protein have evolved. He said the landscape has to be smooth in at least some dimensions, otherwise we wouldn't be here, as we are the products of evolution. Some mutations are 'lethal', but many are not. So evolving proteins wander around in this fitness landscape, accumulating mutations and sometimes changing function. Thus the fitness landscape, even though it's mostly empty, is locally smooth in enough dimensions that evolution by gradual change and selection works. Nature has given us all these proteins that are already occupying places in the landscape that are rich in function. What does that mean? It means that if you mutate existing proteins, you can change their sequences and even hope to improve them for new tasks.

Implementing this in the laboratory is simple: you can even do it in a high school laboratory. You can copy the DNA that encodes a protein that nature gave you on a silver platter or scraped from your shoe, under conditions were you control the incorporation of errors. It's easy to make a lot of imperfect copies. Cells then do the hard work: when you put that DNA into microbes, they will start reading it and make proteins that have mutations in them. And you, the breeder of molecules, then have to decide who goes on to parent the next generation, using good old-fashioned analytical chemistry to measure how the proteins behave and choose one(s) that is/ are better than what you had before. You really don't have to look at huge numbers of variants to find improvements, if you've asked the right kind of question. You have to be able to measure the small improvements expected from small sequence changes (1 mutation), and then you iterate on this process in an uphill walk to accumulate beneficial mutations and optimize for a new, evolved feature. 


\section{Rhodothermus marinus cytochrome c makes C-Si bonds}

\section{Gram-negative, themohalo philic bacterium from}

hot springs in Iceland

124 a mino acids, denaturation (melting) temperature $T_{m}=106{ }^{\circ} \mathrm{C}$

\section{Native func tion is electron transfer}

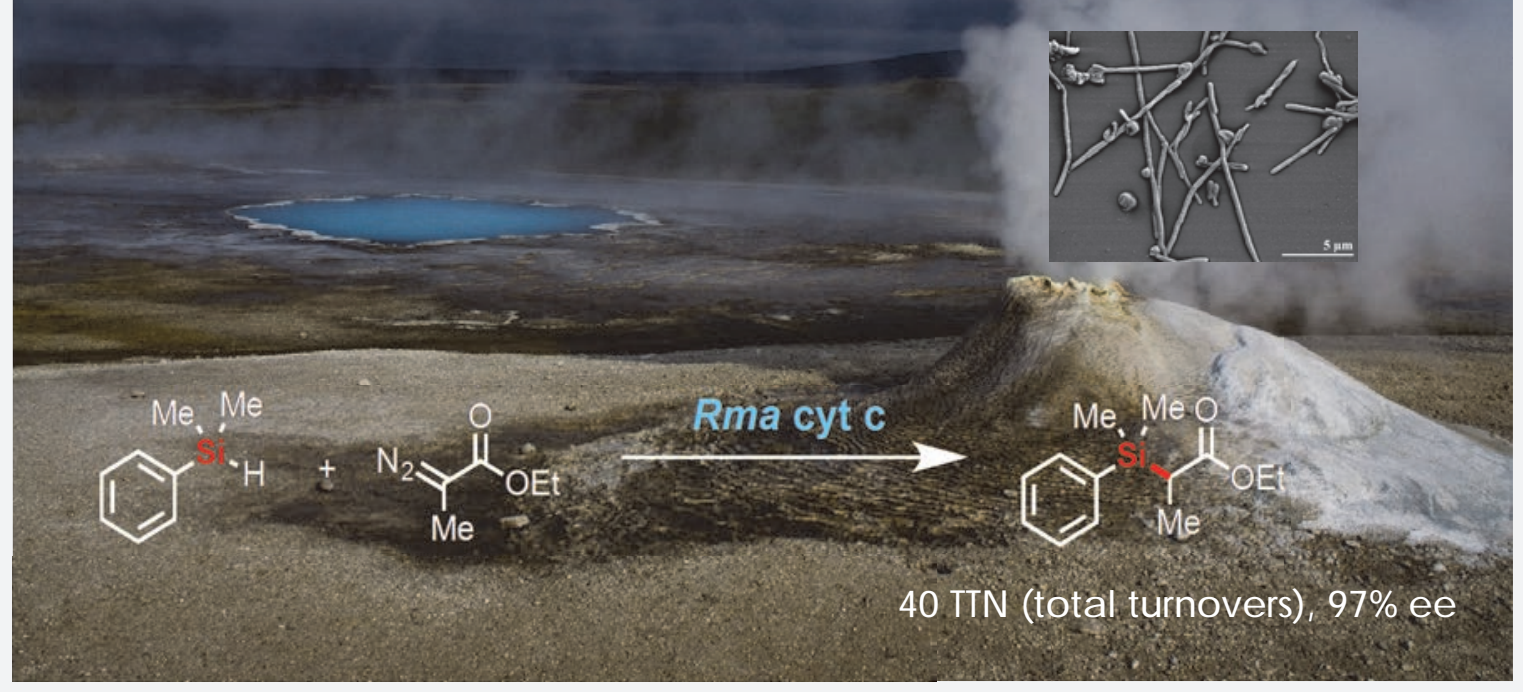

I'm going to quickly summarize the work that lots of people have done in this field before I tell you what is new. Proteins adapt very quickly by this process. It's amazing how well it works, it's the stupidest optimization strategy you can think of - a random uphill walk — but it has generated thousands of useful new proteins that are tuned for what humans would like them to do. The scary fact is that when you try to reverse-engineer the products of this evolution, you find mutations, for example, that are 30 angstroms away from the active side of your enzyme. No one can even explain how those mutations improve catalytic activity, but they do. This just goes to show that we are far away from being able to predict them. Proteins are complicated, but evolution takes care of all of those details. And evolution is great for optimization.

Now, I promised you that I would address the evolution of new chemistry, not just optimization of what nature has already given you. Creating a new enzyme could appear to be a very a hard problem, where you have to perfectly position multiple functional groups simultaneously to catalyze a new reaction. But it's not such a difficult problem if you think about how nature does it. Nature is making new enzymes all the time. l'll just give you two quick examples. We dump potent herbicides like atrazine into the natural environment. We think they are non-biodegradable and then Boom! some organism has found out that if it removes this chlorine atom, it has a rich new nitrogen source that gives it a selective advantage. Then the organism moves all over the world, and atrazine now bio-degrades. All because a soil microbe discovered a new de-chlorinase activity. And it found that activity in an existing, catalytically promiscuous enzyme, whose biological function was something else altogether. Here's another one that you might have seen recent headlines: organisms are learning how to eat plastics that we have been dumping into the environment only for 50-60 years. The organisms and their enzymes are adapting rapidly to take advantage of this new opportunity, although perhaps not rapidly enough.

Also think about antibiotic resistance. Nature is solving all sorts of interesting problems in real time. And how does nature do it? Nature solves problems because the capabilities to do so are already there: novelty is already there. Like enzymes, each one of us has multiple capabilities. Someone may be selected for their ability to work long hours in a laboratory, but they can also play the piano or do dishes if necessary. Enzymes, too, have multiple capabilities that are not being used at a given time. But when a new opportunity arises for it to shine, the enzyme is poised to do it. This new activity becomes beneficial and can be optimized through the random uphill walk process that I just described. 


\section{Directed evolution increased activity 40 -fold in just three generations}

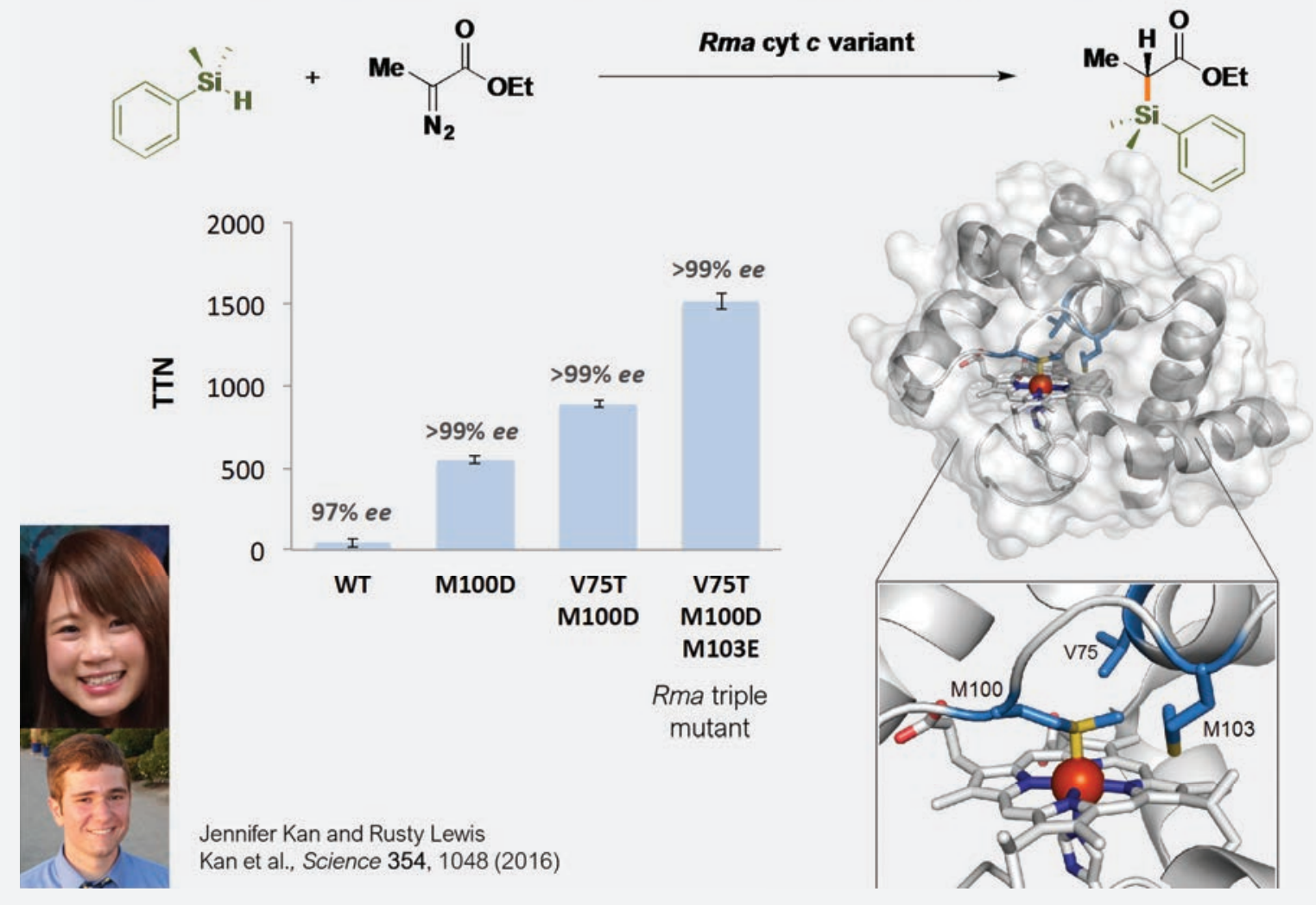

People talk about the Internet of Things. Well, biology is the ultimate Internet of Living Things, a gazillion organisms out there working 24-7, trading genes and crowd-sourcing to solve new problems. It makes sense to use that process to move into the future. But how do I breed forward novelty? How do I use this to create a new enzyme?

A good place to start is with a family of enzymes that has already done it many times. Here is my favorite family, the cytochrome P450s. You have $\sim 50$ of these in your own bodies; they are your first line of defense against various noxious things you ingest. They detoxify chemicals by sticking oxygen in different places and making them easier to excrete or degrade. This enzyme family has gone through mutation and selection not only for optimization of function but also to take on new and different functions. P450s catalyze a whole slew of impressive reactions, from inserting oxygen into un-activated $\mathrm{C}-\mathrm{H}$ bonds, epoxidations, sulfoxidations; they can even nitrate aromatic compounds. This is really powerful chemistry that has all come about through this process of evolution, building on novelty that's already there.

Here's how we thought about the problem of creating a new enzyme. In the presence of what you find in the biological environment and through natural selection, nature built

a mechanism to generate this highly reactive iron-oxygen intermediate in ancient P450. Through divergent evolution, nature has used this ability to generate this reactive species to create all those enzymes that catalyze related, but different reactions. We thought, if we could generate new reactive intermediates, we could then implement human-invented chemistry that uses those. I the breeder could provide a new 'niche' in the form of a synthetic reagent that drives formation of the new intermediate, and then use directed evolution to optimize any new activity that appears. In this way, I should be able to make enzymes that catalyze human-invented reactions that are not known in the biological world.

Here's an example. Chemists have figured out that this iron-porphyrin-carbene intermediate can be used to convert an alkene to a cyclopropane. Or you can insert the carbene into a nitrogen-hydrogen bond and make a new carbon-nitrogen bond. That is interesting chemistry that nature doesn't do, but has been achieved with small-molecule catalysts. We asked: can a heme protein form the iron-carbene intermediate? (There was some evidence indicating that it could: carbenes can be good enzyme inhibitors.) But then instead of transferring the carbene to itself or catalyzing dimerization, could the enzyme transfer it to a second substrate, and could that 


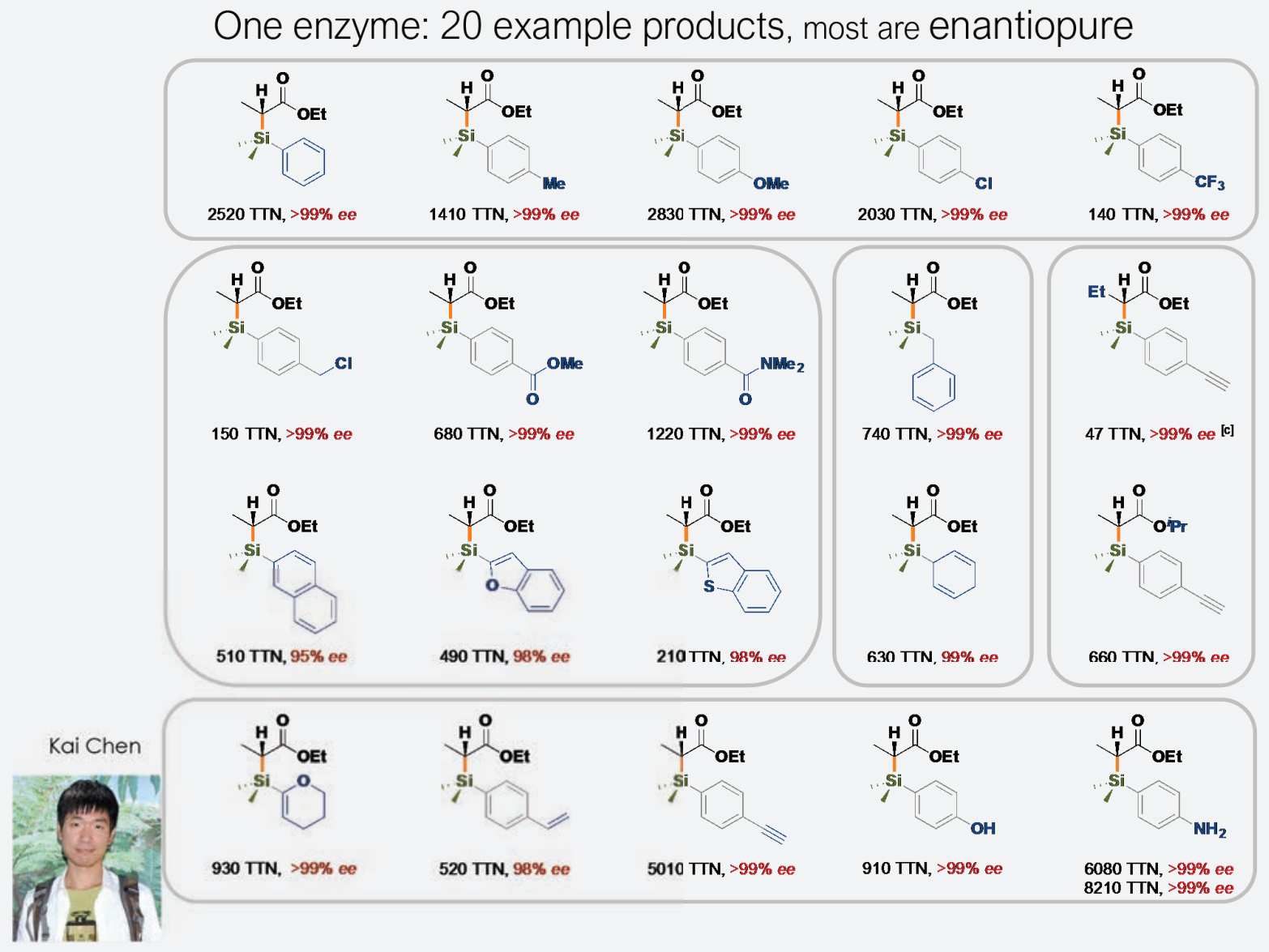

catalyst evolve? The carbene precursors we provide aren't prevalent in the biological world, so nature perhaps never had a chance to do it. But, in fact, if you add styrene and this carbene precursor to various heme-containing proteins, you'll find that they all have a little bit of activity. Even free heme will do it in water. So will cytochrome $c$ and myoglobin. And cytochrome P450 has a miniscule bit of activity. But, remember, for me, that's the starting point for evolution, because the novelty is already there! If I see a little bit of activity, I'm happy, because I can improve it with directed evolution. And, in fact, if you substitute one of the P450's amino acids with another one, activity goes up sixty-fold. Styrene and EDA can make four possible cyclopropane stereoisomers, but this enzyme only makes one. Other mutations allow you to make other products, such as a cis isomer, which is hard to make using small-molecule catalysts.

Now, l'll bring it to life. This new enzyme is encoded in a DNA sequence and should be able to work inside of a cell. Some of you might note that we have dithionite here to reduce the enzyme to its active form, and that's not remotely compatible with a living cell. We try not to contaminate our beautiful evolution experiments with thought, but every once

in a while it's beneficial to do just that. Every P450 on the planet has a cysteine proximal ligand. But these enzymes are so beautifully gated that inside the cell they won't be reduced to the active $\mathrm{Fe}$ (II) form by endogenous reductants without their native substrates. We argued that replacing the cysteine with a serine would change the reduction potential enough that reductants inside the cell would now work. People say, oh that will just kill it. It was well-known that if you make that mutation, it doesn't have any activity. In fact, my original protein is beautiful red, and when you replace the cysteine it turns a sickly green. It's no longer a P450 because it now has its C0-difference peak at $411 \mathrm{~nm}$. (Does that make me the mother of a new enzyme, the cytochrome P411?) Everyone thought that this version was a useless, inactive enzyme, but it turns out that it's now the best cyclopropanation catalyst, or one of the best, ever reported. And it works inside of a living cell. You just use E. coli cells to catalyze the reaction. With seventy thousand turnovers, and the cis product with beautiful enantioselectivity.

It turns out that the P411 is a very special enzyme. It's hard to convey this if you don't have love for some of these molecules, but l'll try to explain. This new enzyme, which 


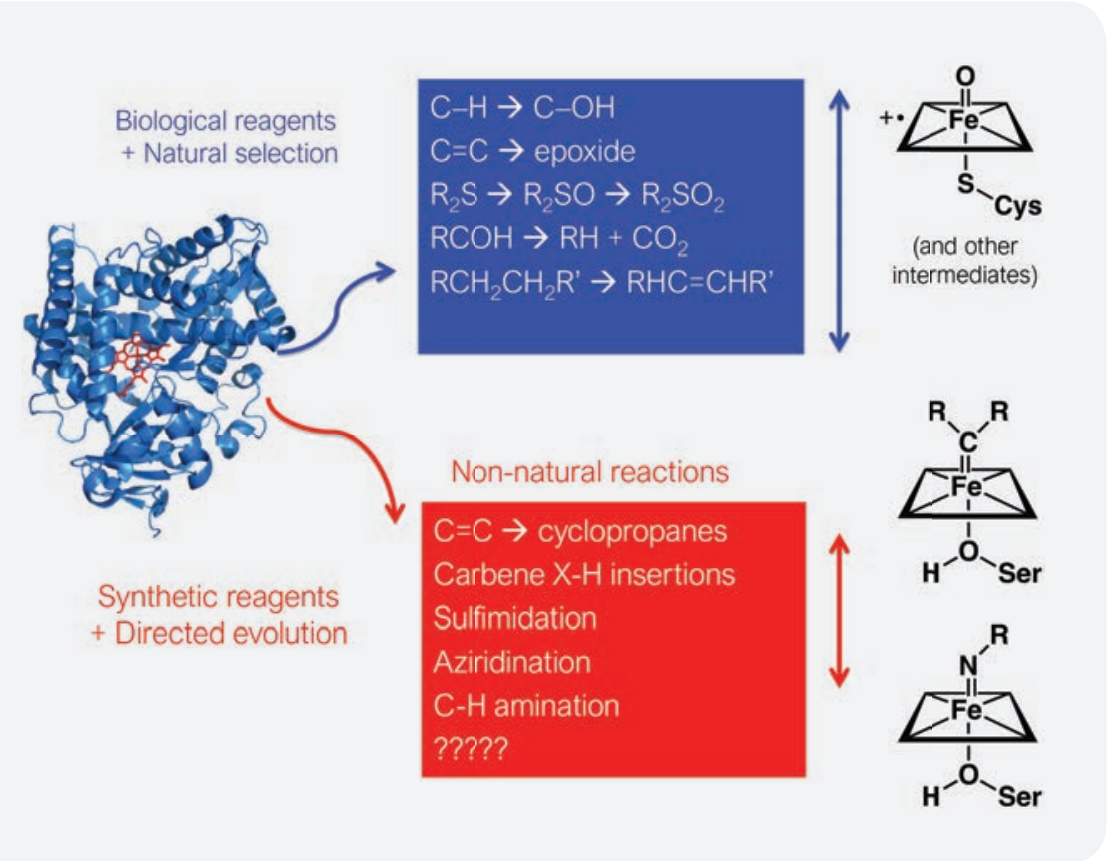

I hope you can now look at a protein with a whole new set of eyes. We see a heme protein as a self-assembling, fully DNAencoded catalyst whose electronic and steric properties can be tuned by evolution. It's an iron complex that can be modified simply by mutation of the DNA to make it capable of all sorts of interesting chemistry. For example, we tuned a P450 to make, in a single step, the cis-cyclopropane core of this FDA-approved drug, Fetzima. You get beautiful selectivity for a single stereoisomer out of the four possible. Another example of great selectivity is the ability to make the key transcyclopropane intermediate to

everybody thought would be dead, is not dead. It's just waiting for you to ask what it can do! For one thing, the P411 can also transfer nitrenes. We tried a lot of enzymes for nitrene transfer, but only found the P411 with this non-natural activity. Two post-docs in my group have shown that you can make a P411 enzyme that catalyzes direct intramolecular $\mathrm{C}-\mathrm{H}$ amination, and the enzyme can react selectively with one of these $\mathrm{C}-\mathrm{H}$ bonds versus another. These two $\mathrm{C}-\mathrm{H}$ bonds are next to one another but differ in their bond strengths by more than $5 \mathrm{kcal} / \mathrm{mol}$. With small-molecule catalysts, you always go to the weak one, and in fact, our first enzyme also did that. But what's really wonderful is that you can use directed evolution to accumulate mutations around the active site that promote reaction at the stronger bond, right next door. That's really impressive, and that's what gets us excited, because we can talk about new chemistry that's really selective.

The next challenge, intermolecular $\mathrm{C}-\mathrm{H}$ amination, is really hard, because the enzyme has to generate the nitrene and transfer it to a second substrate before the nitrene gets reduced. This enzyme has a reductase domain that helps with the initial reduction to the active state, but then also happily reduces the nitrene. So, most of these proteins burn money, so to speak, by reducing the azide. But if you find a little bit of intermolecular amination activity, you can evolve the enzyme and reshape the active site so that this now becomes preferred over the money-burning route. We first demonstrated that you can get intermolecular sulfimidation and aziridination to form these three-membered, nitrogen-containing rings. And just recently we achieved direct (benzylic) $\mathrm{C}-\mathrm{H}$ amination. None of these reactions is known in the biological world, but they're useful synthetic reactions, and they take place inside or outside of cells. another drug, ticagrelor. A single biocatalytic step at room temperature in aqueous solution replaces five or six chemical steps, often involving a precious metal, and provides an environmentally-friendly way to make these drugs.

I'm going to end with one last example, a recent story that you might have seen as it received some attention in the press. Consider silicon. Maybe you've wondered why life does not incorporate silicon into its organic compounds. We find no carbon-silicon bonds in biological molecules, even though silicon is the second most abundant element on the planet's crust. (The most abundant, oxygen, is tied up with a lot of silicon in rocks.) As far as we know, nature has not made enzymes that forge carbon-silicon bonds, or has she? Chemists have invented an array of organosilicon compounds to make sealants, caulks, lubricants, cosmetics and chiral molecules that show up in catalysts, imaging agents, and even LEDs. The history of small-molecule transition metal catalysis for enantioselective carbene insertion into $\mathrm{Si}-\mathrm{H}$ bonds is a short story. There are a few catalysts reported to catalyze carbene insertion, but notice the metals: iridium, copper, rhodium, silver, but there's no iron. Iron has never been reported to catalyze this reaction. And look at the activities: at most one hundred total turnover numbers. Nice selectivities, but the activities are pathetic, and a lot of these have to work at minus 80 degrees centigrade. We wondered whether we could find a protein with activity for this reaction.

By now, we have in my refrigerator at Caltech a good collection of hemeproteins that we can test. Interestingly, it turns out that it is not hard to find activity for forming carbonsilicon bonds, as many hemeproteins will do it at some level. The one I like most, however, comes from a thermohalophile, a bacterium from hot springs in Iceland. This cytochrome $c$ is 
so stable that you can autoclave it! It is like a little rock. It's a small protein too, only 124 amino acids. This protein has no native catalytic activity; its function is electron transfer. In fact, its crystal structure shows a histidine coming up to ligate the iron, and it's got a methionine on the other side as the sixth, axial ligand. No free coordination site! If you think too hard about these things you might not even test it. In fact if you try to calculate the volume of the supposed active site, you find it's zero: there's no active site, at least according to the static crystal structure. If you proposed to test this for catalysis, your professor might say, Ah that's dumb, it obviously won't work. But nature does not care about your calculations. We did test it, and it catalyzes the reaction 40 times. And look at that: 97 percent ee, almost perfect enantioselectivity! That's a remarkable result, just for a native protein; all you have to do is provide these synthetic reagents. And, when you do the directed evolution, targeting this axial methionine ligand and other residues that sit where you think the active site is, you can accumulate beneficial mutations and make catalysts that do this reaction thousands of times, much better than any human chemist has been able to do. A single version of this enzyme can make dozens of new organosilicon compounds. We reported in our paper in 2016 twenty new organosilicon products, 19 of which had never been described before (which of course made dealing with the referees a bit problematic, and we had a supplemental information section describing these things that felt about a thousand pages long with all the NMRs etc.).

l'll just point out a few cool things about this enzyme. There's a double bond on this substrate, and I just told you that if you add carbene precursors, the heme proteins can catalyze cyclopropanation. Or you can insert into the $\mathrm{N}-\mathrm{H}$ bond of this other substrate. And, in fact, the native cytochrome $c$ will generate a mixture of products, with no chemoselectivity. But when you evolve it for silicon-carbon bond formation, you see it strongly prefers insertion into the $\mathrm{Si}-\mathrm{H}$ bond over cyclopropanation or N-H insertion. It 'learns' how to bind the substrate in its active site to make exactly what you screened for. That's what is so amazing about the world of enzyme-catalyzed chemistry: we can get away from processes that make multiple products, one of which you want and the others you don't, because we evolve chemo-, regio- and stereoselective enzymes.

When you look at how this problem was solved, you realize that we are a long ways away from being able to do this by design. With a very high-resolution crystal structure we find that those three mutations have completely remodeled the loop that sits over the active iron atom. Residues on the loop have moved out into the solvent, which carves out a whole new substrate binding pocket. Now, if you roll a little ball over the active site on your computer, you can see that these mutations have generated an active site into which the substrates can fit and where the reaction can happen, better than before.

And it works inside of living cells! The catalyst is fully genetically encoded, nothing fancy about it, no new metals. You just take the DNA encoding these three mutations, put it inside bacteria, and they will do the chemistry happily, for sugar. It may annoy some chemists, who are working really hard to do this in the laboratory, that a bacterium will do it for sugar, but I hope that's the future of chemical synthesis.

When I first told this story, before the paper was published, I couldn't talk to the Science reporter who was sitting in the front row during my talk. Afterwards, he wanted me to tell him more. I said, No, you know I can't tell you because I want to publish it in Science. The reporter still wrote the story and made up whole parts of it! I was delighted when it went all over the world, because his version showed Dr. Spock and Captain Kirk looking for the Horta, life inside of rocks. But that was backwards. I'm not looking for life inside of rocksI want to put rocks inside of life. I want to put silicon into life and start exploring what it can do. What happens when you make silicon-containing lipids or silicon proteins? How do they behave, and where can you go if you can build life from whole new backbones?

The ideas I have presented today are really very simple. This is the great thing about chemistry and manipulating the biological world. We now have the tools and methods that allow us to go out and explore well beyond where nature would ever care to go, and perhaps we can make life come up with new solutions to deep human problems. By thinking a little bit about how nature solves a problem, using evolution, we can now expand nature's chemistry space into new reactive intermediates, new catalysts, and lots and lots of interesting new chemistry.

In my lab, smart young chemists are looking at life in a new way, thinking, Wow, could I do this new chemistry? I think we'll see an explosion of new chemistry that can be catalyzed by the biological world, with just a little help. This powerful design process of evolution doesn't just optimize, it also innovates. New species come up, new catalysts come up, whole new solutions to problems come up from this process of mutation and selection. We can now marry this powerful evolutionary engineering process with human creativity and 200 years of wonderful chemistry, and solve important problems. It's a fun place to be.

I'll thank the young people who did all of this work at Caltech. I get to go around and talk about it, but they do it. It is truly exciting to see how creativity pops up when people combine different experiences, and I hope that I have given you an inkling of where the future can go. 